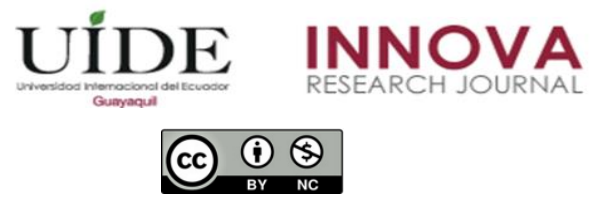

INNOVA Research Journal, ISSN 2477-9024

(Septiembre-Diciembre 2020). Vol. 5, No.3.2 pp. 19-32

DOI: https://doi.org/10.33890/innova.v5.n3.2.2020.1600

URL: http://revistas.uide.edu.ec/index.php/innova/index

Correo: innova@uide.edu.ec

\title{
Aplicación del U-learning en la educación superior del Ecuador frente al COVID-19
}

\section{Application of the U-learning in the higher education of Ecuador against COVID-19}

Gladys Gioconda Lagos Reinoso

(iD) https://orcid.org/0000-0002-9893-1211

Universidad de Guayaquil-Universidad Agraria del Ecuador

Emma Fernanda Garcés Suárez

Universidad de Guayaquil, Ecuador

Autor para correspondencia: gladys.lagos.r@hotmail.com; emma.garcess@ug.edu.ec Fecha de recepción: 06 de octubre de 2020 - Fecha de aceptación: 10 de diciembre de 2020

\section{Resumen}

La tecnología ha cumplido un papel trascendental durante el confinamiento por el COVID-19, permitiendo navegar a través de la disrupción, eliminando fronteras de tiempo y espacio. La presente investigación evalúa la aplicación del U-learning en la educación superior del Ecuador frente al COVID-19, específicamente en la Universidad Agraria del Ecuador, carreras de Medicina Veterinaria e Ingeniería ambiental. Se realizó un estudio del tipo no experimental descriptivo a 140 estudiantes y a 15 docentes. Se aplicó un cuestionario estructurado con 12 preguntas y 5 ítems. Los resultados indican que el $89 \%$ coincidió que este modelo fomenta el trabajo colaborativo, el 94\% consideró que flexibiliza la educación, el 95\% permite un aprendizaje personalizado. Se Concluye que 1 U-learning, tiene un alto nivel de aceptación en la comunidad universitaria, mejora el desempeño individual y colectivo, por lo que es necesario implementar políticas institucionales que permitan su incorporación definitiva en el currículo dentro de la Educación superior del Ecuador.

Palabras claves: aprendizaje; confinamiento; educación; u-learning; COVID-19

\begin{abstract}
Technology has played a vital role during the confinement of COVID-19, allowing it to navigate through disruption, eliminating boundaries of time and space. This research evaluates the application of U-learning in higher education in Ecuador compared to COVID-19, specifically at the Agrarian University of Ecuador, Faculty of Veterinary Medicine and Environmental Engineering careers. A descriptive non-experimental study was carried out on 140 students and 15 teachers. A structured questionnaire with 12 questions and 5 items was applied. The results indicate that $89 \%$ agreed that this model encourages collaborative work, $94 \%$ considered that it makes education more flexible, 95\% allows personalized learning. It is concluded that U-learning, has a high level of acceptance in the university community, improves individual and collective
\end{abstract}


performance, so it is necessary to implement institutional policies that allow its definitive incorporation into the curriculum within higher education in Ecuador.

Keywords: learning; confinement; education; U-learning; COVID-19

\section{Introducción}

La tecnología ha jugado un papel trascendental en la historia de la humanidad justamente en estos momentos en la que un confinamiento era la clave entre vivir o morir. Esto implicó en rompimiento de paradigmas y la reestructuración de estándares y lineamientos existentes antes de COVID-19. Pero fue a partir de este momento en que los seres humanos demostraron su capacidad y poder de resiliencia en la que millones de nuevas oportunidades comenzaron a surgir. Estos cambios afectaron a todos los ámbitos conocidos hasta el momento tanto los netamente tecnológicos como los manuales. Este 2020 será recordado en los libros de la historia universal como una de las épocas más dolorosa que se ha vivido, pero precisamente este estado de confinamiento es el que ha incrementado la dependencia de las personas hacia la tecnología. Son innumerables las actividades en las que intervienen las tecnologías de la información y comunicación, volviéndose en la vida de las personas algo indispensable. Para teletrabajar, seguir clases, hacer deporte, estar en contacto con nuestros seres queridos, combatir el aburrimiento, comprar, etc. Además, las soluciones tecnológicas se han puesto en primera línea para ayudar a combatir los efectos de la enfermedad: desde la movilización de las estructuras para construir respiradores e imprimir equipamientos de protección, hasta la utilización de aplicaciones de inteligencia artificial para buscar los tratamientos más efectivos contra el virus. También se han intentado detectar positivos por la voz, por radiografías de tórax, e incluso se han creado aplicaciones móviles para la trazabilidad de los contagiados. Y es que a través de la disrupción que se ha generado surge una verdadera transformación digital, haciendo que aquellos entornos VUCA Volatility Uncertatinty Complexity Ambiguity (de Toro, 2020), sean el puntal hacia nuevas y mejores formas de hacer las cosas.

La industria de la tecnología avanza a pasos agigantados en un universo que no se detiene, donde cada instante se observa el diseño e implementación de tecnologías que vienen a cubrir necesidades de la sociedad en todos sus aspectos. Y es que, ligado a las grandes necesidades de la humanidad, siempre han aparecido tecnologías de gran importancia cuyo enfoque se torna universal al hablar de comunicación como el teléfono, la televisión, la radio, internet, en el campo de la salud, han surgido avances en la detección, tratamiento y búsqueda de la cura para el coronavirus. También, a nivel económico han surgido nuevas tecnologías industriales, tecnologías de procesos, de extracción. Con el confinamiento surgió la oportunidad de hacerlo todo sin restricciones de tiempo ni espacio adaptando ese espacio de la sala, dormitorio o biblioteca de forma personalizada ajustada a su reciente cambio de estilo de vida, por lo que el papel fundamental de la tecnología en los actuales momentos reside en el impacto que genere en la sociedad a este proceso lo que se le llama revolución tecnológica a base del Covid-19.

En este nuevo panorama se ve inmersa la educación y su relación con la tecnología. A lo largo del tiempo se ha hablado de las ventajas educativas que aportan la formación en línea y sus

Esta obra se comparte bajo la licencia Creative Common Atribución-No Comercial 4.0 International (CC BY-NC 4.0) Revista de la Universidad Internacional del Ecuador. URL: https://www.uide.edu.ec/ 
beneficios paralelos. Y es que, en contextos de pandemia se hace emergente una respuesta en línea. Ante una de las primeras medidas de amplio espectro que se aplicó en todos los países afectados del Covid-19, ha sido el cierre de los centros educativos de todos los niveles. Esta la suspensión de actividades en las instituciones educativas ha afectado al 91,3\% del total de estudiantes matriculados en el mundo: más de 1.500 millones de personas se han quedado sin poder asistir a sus clases presenciales. (UNESCO, 2020). El entorno educativo se volcó hacia un aprendizaje en casa pensando en sobrevivir ante un enemigo extremo que no mide edad, clase social ni fronteras. Mas sin embargo a tercera parte de los niños en edad escolar de todo el mundo (463 millones) no pudieron estudiar y muchas escuelas se cerraron, a la educación a distancia cuando la Covid-19 obligó a cerrar sus escuelas (UNICEF, 2020).

El aprendizaje virtual se convierte en el medio para concebir la continuidad de una educación lo más parecida posible a la prespecialidad, claro está que se lo tomó de forma abrupta y sin casi nada de preparación, por lo que realmente no corresponde a un sistema de aprendizaje online o por lo menos no responde a un modelo de aprendizaje o de enseñanza-aprendizaje online. Y allí surge el cuestionamiento interno de cada docente, sin que éste pueda garantizar un aprendizaje de calidad en lo cognitivo, social y didáctico (Garrison et al.,2005), se notó ampliamente como los docentes se enfrentaron a este reto sin precedentes sin contar con la suficientes competencias digitales, más sin embargo nuevamente la vocación y los deseos de servir llevaron a buscar la manera de aprender y empoderarse las herramientas digitales necesarias para enfrentarse a esta transformación de los procesos de enseñanza aprendizaje en medio de una pandemia voraz.

\section{Marco teórico}

Las organizaciones Unicef y Unesco resaltaron que, en contextos de emergencia, como la que está viviendo el país frente a la pandemia Covid-19, los Estados deben hacer su máximo esfuerzo para velar por el acceso universal y sin discriminación a la educación de los niños, niñas, adolescentes y adultos que estudian. Sin embargo, al inicio de estos duros momentos, la comunidad en general no podía asimilar que todo el entorno educativo ha cambiado, y que debe enfrentar una nueva realidad, algo en lo que nadie jamás pensó se dio. Y aunque muchos pensaban que la emergencia sanitaria no duraría más de un par de semanas, la situación se complicó a nivel mundial y el confinamiento siguió alterando todas las rutinas existentes en el mundo.

En este cambiante mundo tecnológico el manejo y desenvolvimiento de los diferentes entornos puede estar lleno de experiencia, planificación y desarrollo frente al manejo de plataformas virtuales, y recursos que están específicamente diseñadas para impartirse online (Hodges et al., 2020). Todo este gran esfuerzo que tanto docentes como estudiantes están llevando a cabo en sus salas de clase virtuales se lo ha dado como respuesta de emergencia sin tener el tiempo para realizar un rediseño de carreras impartidas y cursadas de forma presencial (o semipresencial en el mejor de los casos), hacia un mundo ubicuo, en medio de un universo de limitaciones que van desde el acceso a internet, hasta la infraestructura tecnológica deficiente con que cuentan en más de un establecimiento educativo, que todos los actores contaban con los medios tecnológicos mínimos requeridos, las competencias digitales necesarias y unas actitudes proclives al cambio. Durante este tiempo se ha puesto de manifiesto la existencia de tres brechas 
(Fernández Enguita, 2020), muy bien marcadas, una brecha de acceso, relacionada con tener o no tener acceso a dispositivos electrónicos y/o a conexión a Internet, la brecha de uso, relacionada con el tiempo de uso y la calidad, es decir hogares en los cuales solo existe un dispositivo y este es compartido por todos los miembros de esa familia, y por último la brecha de competencias, relacionada con las competencias digitales del docente y del estudiante para utilizar adecuadamente las plataformas digitales con fines educativos y la capacidad de elaborar y manejar recursos educativos digitales. Se debe considerar también que la forma de evaluar ha cambiado por tanto el docente debe renovarse y entender que el entorno es totalmente nuevo, sin querer pretender evaluar como lo hacía en modo presencial ya que las variables de evaluación han cambiado. Incluso la forma de hacer los talleres y trabajos en clase han variado pudiese hacerlo en modo virtual a través de las plataformas digitales formales e informales como Facebook, Twitter hasta YouTube. Las herramientas educativas digitales son muy variadas y están disponible en la web de forma gratuita y otras pagadas, y estas ayudan a los estudiantes a obtener la información necesaria que luego en las jornadas en línea con el docente se podrá transformar esa información en conocimiento. Contar con el equipo adecuado que ayuda a abrir caminos para la actividad de los estudiantes, ayuda a procesar y movilizar las ideas y conecta esas ideas con los contextos que las rodean y constituye la base del entorno de enseñanza y aprendizaje.

Antes de Covid-19 el acceso a la educación superior era insuficiente para los miles de jóvenes que deseaban ingresar a las universidades públicas la gran mayoría en su modalidad presencial, con todas las limitantes que esto conlleva, pues para muchos de los futuros universitarios no es opción tener que abandonar su trabajo por el estudio, siendo más fácil que abandone sus estudios que su trabajo que muchas veces de ese sueldo depende toda una familia. Ese era el panorama más frecuente escases de cupos para el ingreso a la educación superior pública, falta de recursos económicos y eso se le agrega varios factores como las distancias geográficas entre los centros de estudio y el lugar de residencia, por lo que siempre se planteó la necesidad de, iniciar una verdadera transformación digital que obligaba a replantear los modos de actuar y relacionarse y ya existía ese anhelo de aplicar un aprendizaje libre de restricciones de horarios y espacios.

Ahora durante el Covid-19, el Aprendizaje ubicuo toma fuerza y es que el concepto de ubicuidad en todos los campos se ha hecho presente durante la pandemia, el teletrabajo es un ejemplo de la importancia de esta metodología y es que a más de uno le tocó trasladar sus negocios a la web donde no existe el concepto de fronteras geográficas ni de límites. El Ulearning permite vencer los limitantes existentes en la actualidad producto del confinamiento y distanciamiento social, las ventajas siguen siendo las mismas que en todas áreas de su aplicación en términos de libertad en tiempo, ahorro de recursos y sobre todo no hay movilidades. La universidad debe aspirar a que los estudiantes reciban la educación a la que tienen derecho, con independencia de sus capacidades, sexo, condición social, económica o de cualquier otro tipo.

Según la UNESCO, Organización de la Naciones Unidas para la Educación, la Ciencia y la Cultura, la educación inclusiva y de calidad se basa en "el derecho de todos los alumnos a recibir una educación de calidad que satisfaga sus necesidades básicas de aprendizaje y enriquezca sus vidas". El artículo 26 de la Declaración Universal de Derechos Humanos de 1948 recoge el derecho de cada individuo a la educación. Cuando se habla del U-learning Aprendizaje 
Ubicuo se lo relaciona con el aprendizaje situado (Lave et al., 1991) teoría general de la adquisición de conocimiento donde se explica al aprendizaje verdadero dentro del contexto de actividades de la vida cotidiana, donde el aprendizaje formal en el aula implica la abstracción del conocimiento y la descontextualización a favor del proceso cognitivo. El aprendizaje ubicuo es algo característico del ser humano, el aprendizaje llega en todo lugar y en todo momento, evoluciona y se adapta al entorno como lo menciona Vygotsky en su teoría del aprendizaje basado en el aprendizaje sociocultural de cada individuo y en el medio que se desarrolla. De allí que al cambiar el entorno readecua su aprendizaje al momento en el que se encuentra. En una formación ubicua se enlaza el aprendizaje y la tecnología ubicua dentro de modelo pedagógico U-learning acompañado de las tecnologías de la información y comunicación, el internet, las redes sociales, por lo que ya no requiere de un espacio físico si no que está abierto al tiempo y espacio de cada estudiantes, este aprendizaje situacional va a introducido se da en el contexto de situaciones o problemas que el individuo enfrenta y donde se aplicará lo aprendido en relación a ese problema que de forma específica se presenta. (Burbules, 2009).

Esto conlleva a un aprendizaje dinámico y personalizado que bien puede ser formal o informal para luego constituirse en un aprendizaje colaborativo que tiene lugar en un contexto social y el significado o contenido de los nuevos conceptos, constituyen una forma de generar esquemas cognitivos llevando el proceso de enseñanza aprendizaje al plano social en la que el aspecto pedagógico mediante una comunicación eficiente y el acceso a los recursos en línea. El aprendizaje ubicuo, se produce a partir del entorno, el ser humano está en constante aprendizaje y recibe los estímulos que están a su alrededor de allí que en medio de este nuevo panorama de confinamiento se desarrolla una nueva forma de enseñar y aprender en función del nuevo entrono que se tiene, esto va a permitir enriquecer el conocimiento al relacionar esa empatía docente estudiante hacia la asignatura y la tecnología ubicua lo que va a permitir conceptualizar importantes avances.

Dentro de lo ubicuo esta lo móvil y los celulares encasillan en esa selección, Zapata Ros (2012), señala que el aprendizaje móvil o ubicuo es el aprendizaje apoyado por la tecnología o del aprendizaje conectado. En este contexto los dispositivos móviles son coadyuvantes de la ubicuidad en el proceso de enseñanza aprendizaje siendo los celulares un dispositivo que se pueden llevar en todo momento, son de fácil uso y es más probable que una persona tenga un celular que un computador, por lo que su presencia se vuelve imprescindible en la cotidianidad.

En educación existe mucha creatividad de parte de los docentes quienes están usando todas las modalidades posibles de comunicación y enseñanza tratando de acomodarse a la infraestructura existente en su entorno y en el de sus estudiantes. Entrega utilizando la infraestructura existente. Usar herramientas en línea para asegurar que las guías pedagógicas con planes de clase detallados, videos, tutoriales que los mismos docentes están creando en YouTube, para sus propios estudiantes y probablemente serán usados por todos. También se está usando el podcast y otros recursos que requieran menos uso de datos al bajarlos al celular siempre que este sea inteligente. Hay países en los que están también habilitando sus plataformas de educación a nivel de la radio y la televisión también son herramientas muy poderosas. La ventaja que tenemos hoy en día es que, a través de las redes sociales, WhatsApp o SMS, lo que al menos puede garantizar una comunicación rápida efectiva y directa dentro del proceso de aprendizaje, utilizando contenido entregado por radio o TV. El aprendizaje ubicuo hoy es ya un 
aprendizaje digital integrado donde convergen las plataformas formales e informales, con único propósito de que el estudiante aprenda y no se vea detenido su progreso y desarrollo como miembro base de la sociedad.

En medio del caos provocado por el COVID-19 es precisamente este aprendizaje ubicuo el que aporto y está aportando en las planificaciones y estrategias de los docentes, a fin de brindar oportunidades de aprender a los estudiantes que no dispongan de computador, y que a través de un enlace móvil pueden acceder a las charlas en línea, la biblioteca además de que pueda ser evaluado, bien sea desde su aula virtual o desde cualquier red social donde se compartan las actividades curriculares y experiencias de la clase, lo que al final permitirá un aprendizaje incluyente y acorde a la realidad social que se está atravesando. Se habla entonces de que los estudiantes tienen la libertad de aprender dentro de un ambiente de aprendizaje personalizado y adaptable a las necesidades individuales y a sus estilos de aprendizaje (Jones y Jo; 2004:469).

Otro tipo de aprendizaje ubicuo está en el concepto del manejo de datos en la nube (Clound Computing) volviéndose omnipresente aportando seguridad, confiabilidad y escalabilidad con un diseño orientados a maximizar la eficiencia, en el desarrollo aplicaciones en la nube que luego se pueden escalar de manera mucho más poderosa. El informe Horizon en el 2010 menciona a la computación móvil, la realidad aumentada y de la computación basada en gestos, con múltiples usos. En el año 2012, este informe incide en elementos que van mucho más allá de la tecnología: las oportunidades para el aprendizaje informal, poder estudiar y trabajar dónde y cuándo quieras; el cloud computing; el trabajo colaborativo; el cambio que supone internet para la educación, etc. (De Castro Lozano, 2012)

Finalmente, se tiene la masificación del aprendizaje, donde se está haciendo referencia a una educación abierta y sin limitaciones donde cualquier persona tendrá acceso a la educación en cualquier parte y a cualquier hora, abriendo el espacio para la inclusión de cientos de personas que hasta hace poco veían frustrados sus deseos de aprender.

Sin embargo, a todo esto, se debe considerar que, pese a que siempre se ha mostrado las bondades de un aprendizaje ubicuo, es durante esta emergencia sanitaria mundial, que se permitió desnudar un sistema educativo deficiente en cuanto a infraestructura tecnológica y competencias digitales, por lo que entra en juego cuan capacitados están los docentes y estudiantes para adoptar de una manera tan abrupta este nuevo modelo de enseñar y aprender.

Hoy el papel del docente es de gran trascendencia por lo que será la base donde se va a soportar todo este nuevo entorno, por lo que entra en elevada significancia la innovación y creatividad en cuanto al uso y manejo de herramientas pedagógicas digitales en las diferentes aulas virtuales, lo que permitirá la reactivación del sector educativo para enfrentar este nuevo reto con responsabilidad social, lo que conlleva a la construcción de los distintos escenarios (Castañeda et al., 2020).

Este sistema de educación ubicua va más allá de digitalizar contenidos, o de sustituir la hora de clase presencial en el aula por otra hora de clase síncrona en una sala virtual con una herramienta de videoconferencia o enviar material de lectura, entre otras alternativas, se trata de 
hallar la manera de establecer esa empatía necesaria para construir el conocimiento. Se tiene que rediseñar la experiencia de aprendizaje (Llorens-Largo, 2020a) y poner en el centro de la formación online la interacción (García-Peñalvo y Seoane-Pardo, 2015; Seoane-Pardo y GarcíaPeñalvo, 2014), entre docente y estudiantes. Esta nueva modalidad ubicua a nivel mundial busca lograr una verdadera transformación digital que vaya más allá de la simple digitalización de contenidos. (Llorens-Largo, 2020b).

Es precisamente en estos momentos en que las universidades de todas las latitudes están transfiriendo lo material a un formato digital, desde un simple registro de clases hasta una exposición magistral, dentro de un campo inexplorado de la virtualidad. Los docentes y estudiantes tienen un sin número de interrogantes sin respuestas, frente a un momento doloroso para los que la humanidad no estaba preparada, fueron muchas las universidades en las que su modalidad fue siempre presencial dado la naturaleza de sus especialidades y que ahora de la noche a la mañana tuvieron que hacerle frente a la única alternativa para no cerrar sus puertas, entrar en el mundo de la ubicuidad en todos los procesos de enseñanza aprendizaje.

Sin embargo, la realidad es dura de afrontar frente a escenarios donde la situación de salud personal y familiar es delicada, donde miembros de una misma familia han muerto por COVID-19, y otros han perdido sus empleos por reducción de personal o porque muchas empresas y negocios pequeños han quebrado. La deficiente infraestructura tecnológica, la falta de recursos económicos y la escasa inversión de los gobiernos de turno para educación salud y tecnología hace ver un panorama nada alentador ni motivante para la educación virtual. Existen estudiantes que se encuentran alejados de las ciudades donde su nivel de conectividad es escaso junto con la falta de dispositivos electrónicos que le permitan conectarse a sus clases virtuales y que muchas veces si este dispositivo existe debe ser compartido con todos los miembros de una misma familia que estudian y hacen teletrabajo e incluso varios de ellos tienen que movilizarse hasta un lugar alto para tener señal de internet y recibir clases. Y estas escenas no solo es del lado de los estudiantes sino de los docentes quienes tampoco tienen conectividad ni mucho menos los dispositivos tecnológicos necesarios, sin olvidar del escaso dominio de las competencias digitales. Por lo que esta investigación tiene como objetivo analizar la aplicación del U-learning como nuevo modelo pedagógico en el COVID-19 la educación superior del Ecuador.

\section{Metodología}

Partiendo de una necesidad de darle continuidad a la educación en medio de esta crisis sanitaria mundial que ha llevado a toda la población a un confinamiento forzado, las autoridades de la Universidad Agraria del Ecuador, dentro del plan de emergencia activado para retomar las clases en todas las facultades, establecieron un cronograma de capacitaciones constantes dirigido a todo el personal docente de la institución, sobre el manejo de varias herramientas de comunicación digital tales como Zoom, y Microsoft Teams. El inicio de las clases bajo esta nueva modalidad virtual quedó establecido para el 4 de mayo de 2020, dejando en la libertad a los docentes para escoger la plataforma para las clases sincrónicas Zoom y Microsoft Teams apoyados de Edmodo en algunos casos para la parte asincrónica. 
En lo que respecta a la asignatura de Ofimática, del primer semestre Medicina Veterinaria e ingeniería ambiental, las clases sincrónicas se las ha realizado en Zoom, la docente adquirió la licencia pagada para no depender de los 40 minutos que brindan las sesiones gratuitas de esta plataforma. Así mismo se escogió Edmodo, que es una plataforma tecnológica, social, educativa y gratuita que permite la comunicación entre estudiantes y docentes en un entorno cerrado, siendo de fácil uso y entendimiento. Adicionalmente se emplearon plataformas sociales como Facebook, YouTube, WhatsApp para establecer múltiples canales de comunicación que no todos los estudiantes disponen de los recursos tecnológicos necesarios para continuar sus estudios bajo la modalidad virtual.

Según estudios hechos por el Instituto Nacional de Estadísticas y Censos del Ecuador (INEC, 2016) 9 de 10 hogares disponen de un teléfono celular, Donna Abernathy, en el artículo "Get Ready for M-Learning"(2001), alerta sobre el potencial de este tipo de aprendizaje.

Abernathy observa que el m-learning no trata de sustituir el PC, sino que ayudará a complementar los objetivos de aprendizaje de las empresas con herramientas instantáneas. Entre 2002 y 2006, comenzaron a aparecer numerosos estudios indicando que las tecnologías que soportan el m-learning se están expandiendo y que comienzan a ser comunes en una gran variedad de entornos de aprendizaje.

Es por ello, que durante este estudio se trató de buscar alternativas que permitan hacer uso de los teléfonos inteligentes, tablas o un ordenador común con acceso a internet y seguir asistiendo a las clases tanto sincrónicas como asincrónicas, así se diseñó el aula virtual en EDMODO, donde se crearon cursos con tópicos de la asignatura de Ofimática, con varios ejercicios digitales, juegos en línea, test virtuales, se usó Educaplay, Quizizz que sirvieron tanto para aprender, reforzar y evaluar los conocimientos adquiridos bajo esta nueva modalidad. Así mismo en el aula virtual se subieron los enlaces hacia las clase grabadas diariamente para que aquellos estudiantes que no disponían de un plan de datos o megas para poder seguir las clases sincrónicas, lo pueda revisar luego en el omento que consideren pertinente y así no quedarse atrasado, de esta manera se motiva al estudiante a no alejarse de sus aulas de clases y continuar con sus estudios sin importar en qué lugar se encuentren reduciendo las brechas geográficas y de tiempo, donde el acceso a la plataforma se puede llevar a cabo desde cualquier dispositivo móvil e incluso computador portátil por lo que estas herramientas pueden ser aprovechadas por todos los estudiantes sin excepción.

La población de estudio estuvo formada por 140 estudiantes de primer semestre de medicina veterinaria e ingeniería ambiental y 15 docentes. Se aplicó una encuesta de satisfacción al finalizar el primer parcial con 12 preguntas y 5 ítems del tipo abiertas y cerradas, con opciones múltiples jerarquizadas, con las que se evaluó la percepción general, grado de aceptación, por parte los estudiantes al hacer uso de este nuevo modelo de aprendizaje ULearning, también se evaluó el nivel de preferencia entre las clases virtuales y las presenciales.

En cuanto al tratamiento de los datos se utilizó el programa estadístico open source R, en la comparación de los dos grupos de trabajo se realizó a través de T Student para grupos dependientes con un nivel de significancia del 0,05 para rechazar hipótesis nula. La confiabilidad y consistencia del instrumento fue evaluado a través del análisis de covariancia de ítems 
mediante el Coeficiente de alfa de Conbrach, demostrando consistencia y validez en el instrumento aplicado.

Para el análisis y evaluación del rendimiento académico se trabajó mediante la observación y el método histórico, haciendo uso de las actas de calificaciones del semestre pasado referentes al primer parcial procediendo al análisis comparativo para la evaluación de los resultados en base a nivel de satisfacción, aceptación, y rendimiento académico.

\section{Resultados y discusión}

Como resultados de la aplicación del instrumento de evaluación, se puede observar que el perfil general de los 140 estudiantes y 15 docentes encuestados muestra una población equitativa en cuanto al género, ya que el $42 \%(n=59)$, pertenece al género masculino y el $58 \%(n=81)$, para estudiantes y de $48 \%$ masculino y $52 \%$ femenino para los docentes encuestados. Tabla 1 . En cuanto a la edad promedio estuvo dada entre 18 a 22 años, para estudiantes tratándose de un grupo joven, reconocidos como nativos digitales, Prensky (2001), para quienes el uso de las herramientas tecnológicas como los dispositivos móviles y las redes sociales son algo sencillo y común. Mientras que los docentes están relativamente en los inmigrantes digitales ya que sus edades fluctúan entre los 33 y 54 años.

\section{Tabla 1}

Distribución por género

\begin{tabular}{lllllll}
\hline Género & Estudiantes & Porcentaje & Docente & Porcentaje & Total & Porcentaje \\
\hline Hombre & 59 & $63 \%$ & 7 & $47 \%$ & 66 & $42,58 \%$ \\
Mujer & 81 & $38 \%$ & 8 & $53 \%$ & 89 & $57,42 \%$ \\
Total & 140 & $100 \%$ & 15 & $100 \%$ & 155 & $100 \%$ \\
\hline
\end{tabular}

Fuente: Gladys Lagos Reinoso 2020

$\mathrm{Al}$ pedirle que señalen las ventajas del u-learning frente al aprendizaje presencial los encuestados escogieron más de una ventaja mostrado así la gama de beneficios que perciben en la aplicación de este modelo, siendo los más seleccionados el hecho de que no se requiere movilización con el 96\%, lo que les representa horro de tiempo y recursos, por lo que cualquier espacio puede trasformase en un entorno ideal de aprendizaje ubicuo, (University of Illinois, 2009). También tuvo alta selección la flexibilidad con el 94\%, seguida del $80 \%$ en aprendizaje personalizado, ya que este modelo permite que cada estudiante se acomode a su tiempo y espacio pudiendo asistir de modo sincrónico o asincrónico revisando grabaciones o ejercicios luego de la clase, lo que permitirá aprender y reforzar los conocimientos, dependiendo de sus necesidades particulares (Graf, 2008), el aprendizaje autónomo con el 48\%, por lo que dependerá mucho de la disciplina y de la responsabilidad de cada estudiante la continuidad de sus estudios. Figura 1. 


\section{Figura 1}

\section{Ventajas del U learning}

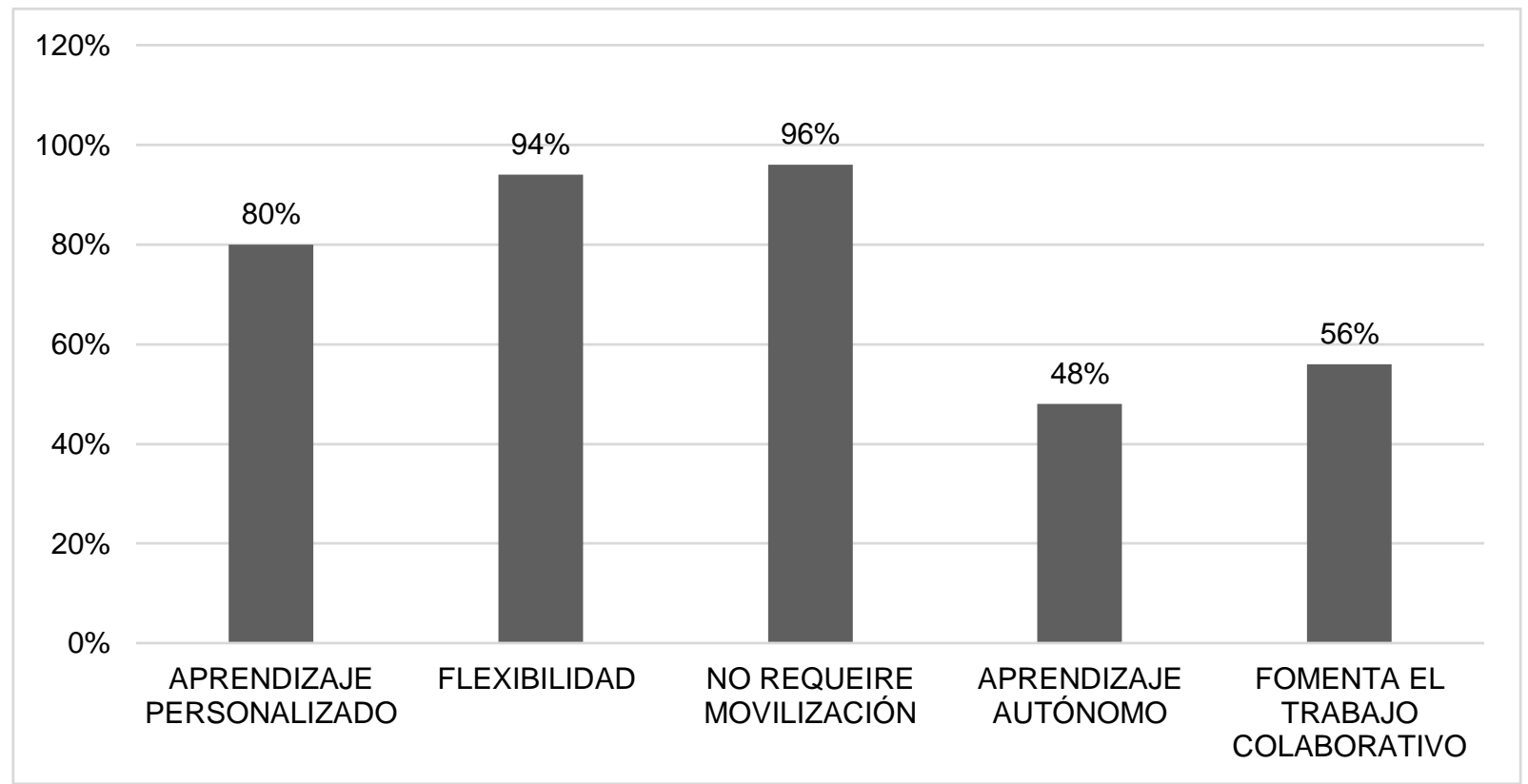

Nota: Se observa los diferentes beneficios obtenidos en el U-learning

En cuanto a los problemas Figura 2 que han tenido que enfrentar los encuestados durante la aplicación del u-learning, se observó que el 87\% considera que el acceso a internet representa un problema serio tanto para docentes como para estudiantes al momento de participar en la educación virtual (Zhang et al, 2004). El no tener un dispositivo electrónico para las clases virtuales obtuvo un $76 \%$, esta es una problemática que afecta a todos los estudiantes y docentes al momento de conectarse a sus clases virtuales, (Bustos ,2005), tan solo el 25\% coincidió que hay escasa interacción a través de la virtualidad. Para lograr esta rescatar la interacción digital entre los actores de este nuevo modelo será adoptar estrategias de orden participativo, con acento en la interacción y la motivación (Brito, 2010). Tanto docentes como estudiantes requieren cambiar los hábitos de interacción y acercamiento al conocimiento, pues hay que desarrollar una serie de habilidades, tanto en el orden tecnológico como pedagógico, que permitan incluir diversas estrategias de formación basadas en la ubicuidad. 


\section{Figura 2}

\section{Problemas presentados en el U learning}

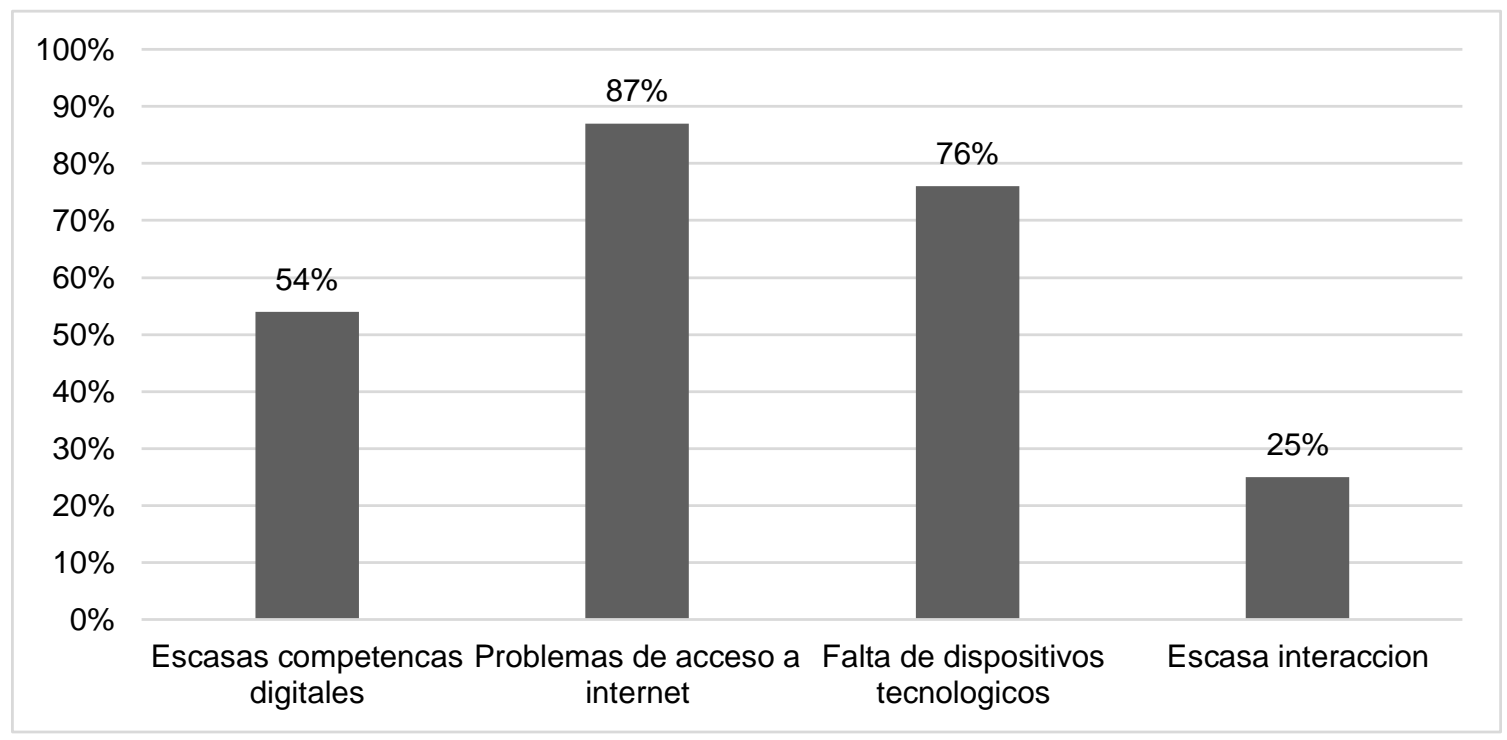

Nota: Se observa los diferentes inconvenientes para hacer uso del modelo U-learning

Mediante la puesta en marcha del modelo U-learning se notó mejoría en las calificaciones del primer parcial lo que repercutió en un incremento favorable del $85 \%$, en cuanto al rendimiento académico, ya que los estudiantes a través de las clases virtuales pueden tener mayor control del material de la clase y existe una comunicación directa con la docente, adicionalmente que a través de las clases grabadas se pudieron reforzar conocimientos a su ritmo y tiempo. y a la vez trabajaron en talleres e investigaciones de modo sincrónico y asincrónico, con ayuda de tutoriales virtuales y el empleo de las plataformas digitales (Facebook YouTube) a más de los grupos de mensajería instantánea que se formaron a la par. Siendo el promedio de respuesta del 4,93 ( $\mathrm{p}>0,05)$. Respecto a la interacción, a nivel presencial fue de 4,81 y a nivel virtual de 4,64 ( $\mathrm{p}>0,05)$. Respecto a la satisfacción general de esta metodología y el desarrollo del curso el $89 \%$ coincidió que esta modalidad brindó igualdad de oportunidades en la educación para todos los estudiantes, Figura 5. El 95\% consideró que su aplicación permite reforzar conocimientos adquiridos además que el $98 \%$ de los estudiantes y docentes lo consideró como una metodología que motiva y estimula el aprendizaje colaborativo.

Los investigadores han explorado el impacto del uso dispositivos tecnológicos en la educación sean computadores, celulares, tabletas, entre otros, en una serie de situaciones hasta la fecha, sobre todo en ensayos a pequeña escala (Glicksman, 2011; García et al, 2011). Sin embargo, existe una coincidencia considerable en los resultados de estos estudios, los cuales, además de destacar los beneficios considerables de su uso, también ponen de relieve una serie de cuestiones técnicas, pedagógicas y de gestión que las escuelas tienen que resolver. Los resultados del estudio en la Academia Longfield, utilizando metodologías de investigación similares, sirven para confirmar aún más el potencial de estos dispositivos como herramientas para el aprendizaje ubicuo (Heinrich, 2012). 
Si bien es cierto, estas herramientas de comunicación digital y de recursos interactivos ya existían hace algún tiempo, no eran usadas en su totalidad en la modalidad presencial como el caso de YouTube Live, Facebook Live donde también se puede transmitir en vivo, logrando incrementar la motivación y el interés de los estudiantes lo que va a permitir aplicar nuevos estilos de enseñanza que beneficien su aprendizaje (Torres et al., 2011). Las posibilidades pedagógicas de estas herramientas son amplias, en las prácticas universitarias se da en diversas formas, a través de grupos o foros virtuales aplican la tecnología en mayor o menor nivel, En un contexto donde las habilidades y la familiaridad de los estudiantes con las nuevas tecnologías a menudo superan las del propio docente, donde su papel sigue siendo crucial y cambiante de magistral a facilitador o tutor. La participación del docente se puede relacionar con uno o varios de estos niveles: espacio personal, aula virtual, entorno virtual de enseñanza-aprendizaje, campus virtual y plataforma (Bautista et al, 2006).

Por lo que educar en tiempos de pandemia se vuelve un verdadero reto que va de la mano de las competencias digitales. Es esta la razón de que varias universidades a lo largo del mundo se estén plateando la posibilidad de lanzar sus carreras en línea de modo permanente algo que desde hace mucho tiempo la sociedad lo viene requiriendo. Se trata de generar a través de una disrupción en la educación tradicional, que genere nuevas oportunidades de acceso en condiciones de equidad a dichas tecnologías, sino también en relación a sus usos pedagógicos, las TIC contribuirían a generar un nuevo elemento de desigualdad, seguido de exclusión social y educativa. (Díaz -Barriga, 2010).

\section{Conclusiones}

El Covid-19 está dejando lecciones de vida de alto alcance y es que no se puede dejar de lado la inversión en educación y salud, dos frentes muy golpeados durante esta pandemia. Por un lado, el desconsuelo por la pérdida de valiosas vidas humanas y por otro esa transformación digital que surge de los procesos disruptivos en el marco de la ubicuidad en todos los procesos alrededor del mundo. Sin embargo, es importante recalcar que si bien es cierto todo fue realizado con la premura e inexperiencia en el manejo de entornos de aprendizaje en línea, la aplicación del modelo U-learning está permitiendo darles continuidad a las clases en los centros educativos de todo nivel.

La ubicuidad se define más como la característica de estar en todo momento y lugar al mismo tiempo y el aprendizaje ubicuo es algo característico del ser humano, desde sus orígenes evoluciona y se adapta gracias al aprendizaje, es allí que el sistema educativo sigue evolucionado, tanto docentes como estudiantes cuentan con más espacios y herramientas para su formación profesional, donde el lugar, condición social, el tiempo y el espacio ya no son impedimento para prepararse, se trata de una educación inclusiva, libre y flexible adaptable a las necesidades particulares del estudiantes.

De acuerdo con los resultados obtenidos en este estudio, se concluyó que el U-learning tiene un alto potencial de aceptación entre la comunidad universitaria, Su aplicación conlleva la oportunidad de entrar en la era del aprendizaje personalizado, flexible sin limitantes, que mejora el desempeño individual y colectivo, por lo que se vuelve necesaria generar políticas institucionales que regulen la implementación del U-learning, como modelo pedagógico en de la 
Educación superior del Ecuador no solo mientras dure el confinamiento por el COVID-19 sino adoptarlo como una oportunidad de ampliar la oferta educativa y empezar a desarrollar políticas que permitan dinamizar la educación post COVID-19.

\section{Referencias Bibliográficas}

Arceo, F. D. B., Arceo, F. D. B., y Lemini, M. A. R. (2006). Enseñanza situada: vínculo entre la escuela y la vida. McGraw-Hill.

Burbules, N. (2009). ¿Otra mirada sobre las TIC? Colección educ.ar: Uso seguro.

Castañeda, L., Salinas, J. y Adell, J. (2020). Hacia una visión contemporánea de la Tecnología Educativa. Digital Education Review, 37, 240-268 Recuperado de https://revistes.ub.edu/index.php/der/article/view/30136

De Castro Lozano, C. (2012). El futuro de las tecnologías digitales aplicadas al aprendizaje de personas con necesidades educativas especiales. Revista de Educación a Distancia (RED), (32).

De la Torre Cantero, J., Martin-Dorta, N., Pérez, JLS, Carrera, CC, y González, MC (2013). Entorno de aprendizaje ubicuo con realidad aumentada y tabletas para estimular la comprensión del espacio tridimensional. Revista de Educación a Distancia, (37).

De Toro, I. S. (2020). La flexibilidad psicológica como herramienta de adaptación a los nuevos retos laborales. Empoderamiento, innovación y compromiso como palancas para hacer frente a un entorno vuca (Doctoral dissertation, Universidad Pontificia Comillas).

Fernández Enguita, M. (2020). Una pandemia imprevisible ha traído la brecha previsible. Recuperado de https://bit.ly/2VT3kzU.

García-Peñalvo, F. J., y Seoane Pardo, A. M. (2015). Una revisión actualizada del concepto de eLearning. Décimo Aniversario.

Garrison, D.R. y Anderson, T. (2005). El e-learning en el siglo XXI. Investigation \& practice. Barcelona: Octaedro.

Graf, S. (2008, November). Adaptivity and personalization in ubiquitous learning systems. In Symposium of the Austrian HCI and Usability Engineering Group (pp. 331-338). Springer, Berlin, Heidelberg.

Gliksman, S. (2011). What do Students Think of Using iPads in Class? Pilot Survey Results

Heinrich, P. (2012). The Ipad as a Tool for Education. A Study of the Introduction of iPads at Longfield Academy, Kent. Naace.

Hodges, C., Moore, S., Lockee, B., Trust, T. y Bond, A. (2020). La diferencia entre la enseñanza remota de emergencia y el aprendizaje en línea. Revisión de Educause, 27. 
Jones, V. y Jo, JH (2004, diciembre). Entorno de aprendizaje ubicuo: un sistema de enseñanza adaptativo que utiliza tecnología ubicua. En Más allá de la zona de confort: Actas de la 21 a Conferencia ASCILITE (Vol. 468, p. 474).

Lave, J. y Wenger, E. (1991). Aprendizaje situado: participación periférica legítima. Prensa de la Universidad de Cambridge.

Llorens-Largo, F. (2020). Transformación digital versus digitalización. Recuperado de https://bit.ly/2tmYFMr.

Llorens-Largo, F., y Fernández, A. (2020). Coronavirus, la prueba del algodón de la universidad digital. Recuperado de https://bit.ly/2Rm917X.

Prensky, M. (2001). Nativos digitales, inmigrantes digitales. En el horizonte, 9 (5).

Seoane-Pardo, A. M., y García-Peñalvo, F. J. (2014). Pedagogical Patterns and Online Teaching. In Online Tutor 2.0: Methodologies and Case Studies for Successful Learning (pp. 298316). IGI Global.

Torres, C. I., y Alcántar, M. D. R. C. (2011). Uso de las redes sociales como estrategias de aprendizaje. ¿Transformación educativa? Apertura, 3(2). UNESCO (2020). Education: From disruption to recovery. Recuperado de https://en.unesco.org/covid19/educationresponse

UNICEF (2020). COVID-19: Are children able to continue learning during school closures? Recuperado de https://data.unicef.org/resources/remote-learning-reachability-factsheet/

Zapata-Ros, M. (2012). Calidad en entornos ubicuos de aprendizaje. Revista de Educación a Distancia (RED), (31). 